\title{
La traduction n'est pas qu'une traduction. Quelques propos sur la traduction d'une écriture fragmentaire bilingue: Cuvântul nisiparniţă (Le Mot sablier) de Dumitru Tsepeneag
}

\author{
Georgiana LUNGU-BADEA \\ Université de l'Ouest, Timisoara \\ Roumanie
}

\begin{abstract}
Résumé :
Nous nous intéressons à la traduction d'une écriture fragmentaire bilingue. Préoccupé aussi bien par son monolinguisme que par le monolinguisme de l'autre (cf. Derrida 1996 - ici surtout par le monolinguisme du lecteur français censé constituer une sorte de pseudo public-source $\left.{ }^{1}\right)$, Dumitru Tsepeneag transforme le bilinguisme du soi en sujet de création, dans le livre Cuvântul nisiparniţă (paru d'abord en traduction, Le Mot sablier 1984). "Cette appropriation (im)possible devient raison génétique de et dans la création, ensuite dans l'autotraduction »; une " expérience d'écriture » où l'écrivain cultive son bilinguisme et sa bi-culturalité, « et éclaire la traduction d'une perspective au moins double : celle de l'écrivain traduit ${ }^{2}$ et autotraduit, mais aussi celle du traducteur écrivain. " (Lungu-Badea 2008, 20). Quelle stratégie de traduction mettre en œuvre pour ce livre qui commence en roumain et se termine en français ? On dirait que sa destinée est de montrer comment une langue remplace une autre et rend, par la suite, inutile la traduction pour les usagers bilingues en question. Si ce n'est qu'un plaidoyer pour la contretraduction, la traduction française, publiée chez P.O.L. éditeur, ne le conteste pas. Elle n'a pu respecter ni le « vouloir dire psychologique de l'auteur » (Ladmiral 2006, 140), ni le « vouloir-dire sémantique du texte »(Ladmiral et Lipiansky 1995, 53).
\end{abstract}

Mots clés : traduction, bilinguisme d'écriture, contre-traduction, écriture fragmentaire bilingue

\begin{abstract}
Translation is not mere translation. A few remarks on the translation of a fragmentary bilingual text: Cuvântul nisiparniță (Le Mot sablier/The Hourglass Word) by Dumitru Tsepeneag

The present paper will focus on the translation of a fragmentary bilingual writing. Interested both in his own monolingualism and in the monolingualism of the other (see Derrida 1996, and here mainly the monolingualism of the French reader who should constitute a kind of pseudo-source-audience3), Dumitru Tsepeneag turns his own bilingualism into a topic in his book Cuvîntul nisiparniță (published first in translation as Le Mot sablier in 1984). "This (im)possible appropriation becomes the generating reason of the creation and in the creation, then in the self-translation"; a "writing experience" where the writer cultivates his bilingualism and his
\end{abstract}

${ }^{1}$ Comme ce fût le cas pour les romans Arpièges et Les Noces nécessaire.

${ }^{2}$ Il s'agit du traducteur, Alain Paruit, auquel le livre est dédicacé.

3 As was the case with the novels Arpièges and Les Noces necessaire. 
biculturalism, and sheds light on the process of translation from a perspective that is at least double: that of the translated $4^{2}$ and self-translated writer, but also that of the translator-writer" (Lungu-Badea 2008, 20). What translation strategy would be appropriate for a book that begins in Romanian and ends in French? We could claim that its destiny is to show how one language replaces another and, consequently, renders translation useless for bilingual users. If this is but an argument for the counter-translation, the French translation, published by the P.O.L. publishing house, does not challenge it. It could respect neither "the psychological intention of the author" (Ladmiral 2006, 140), nor the "semantic intention of the text" (Ladmiral and Lipiansky 1995, 53).

Keywords : translation, bilingual writing, counter-translation, fragmentary bilingual writing

\section{Introduction}

Dans plusieurs de ses œuvres (françaises et roumaines), Dumitru Tsepeneag joue sur le transfert interlingual, sur les limites de la traduction, sur le sentiment de frustration de l'auteur traduit. À ses débuts littéraires en français, il a écrit pour s'y enraciner. Il se trouvait en France au moment où il apprenait sa condamnation à l'exil. Obligé de quitter son pays (en fait, de n'y plus rentrer), accompagné uniquement de sa langue maternelle, il a continué d'écrire en roumain, mais il a publié d'abord en France, ce qui fait que ses œuvres paraissaient d'abord en traduction, n'étant publiées en roumain que des années plus tard. Cette suspension entre deux abîmes ou deux terres fermes, le roumain (langue maternelle) et le français (langue d'accueil), entre deux pays et entre deux langues, deviendra un merveilleux prétexte de création et de fictionnalisation des actes d'écrire et de traduire.

\section{Le deuil de la langue maternelle ou Sur le transfert intralingual et interlingual}

Par le biais de la littérature, Tsepeneag exploite la langue roumaine ou française comme tentative (in)espérée qui lui est offerte, comme à tout être, comme occasion " pour se dire » (Maragnès 1998). Afin de désambiguïser sa réception, de diminuer le risque global de voir s'égarer sa parole dans l'espace de « l'entre deux langues », il fait le récit d'une quête d'identité, pensée et verbalisée grâce au passage du roumain au français ; un inédit exercice littéraire qui illustre la relation qui s'instaure entre l'être et le langage autant que le bilinguisme horizontal et consécutif; l'épreuve de commutation consacre comme langue de création le français qui se

4 By Alain Paruit, the translator to whom the book is dedicated. 
substitue complètement au roumain. ( $v$. dans l'annexe l'explicit libre trilingue). L'insertion graduelle des mots, syntagmes et fragments français, anticipant l'avènement d'un discours entièrement français, n'est pas faite au hasard. Tsepeneag propose aux lecteurs une aventure littéraire et traductionnelle, dans laquelle il enchâsse des remarques relatives au statut du traducteur, un Charon qui fait passer vers l'au-delà de la langue source, un cadavre (de l'œuvre) : la traduction.

Dans Cuvântul nisiparniţă, selon Tsepeneag, il ne s'agit ni de retraduction intrapersonnelle 5 , ni d'autotraduction. Nous ajoutons à la traduction intrapersonnelle un autre déterminant, mentale (Lungu-Badea 2007, 72-86, Bârna 2006, 9-23), qui souligne qu'il s'opère une réflexion en roumain, suivie d'une traduction mentale en français avant de passer à l'écriture en français. Le livre exige ainsi non seulement une lecture filigranée, intertextuelle, mais aussi une contresignature lectorielle (inimaginable, impensable, inanticipable comme la caractérise Derrida 1996), hiérarchiquement subordonnée à la signature de l'auteur, et théoriquement susceptible de récuser les interprétations des contresignataires.

L'auteur comble le narrateur de faveurs lui réservant la tâche de témoigner de ses appréhensions. Depuis qu'il écrit, Tsepeneag suit la quête du soi au moyen de et dans la traduction (allographe, perfective linguistiquement parlant, mais défective, identitairement parlant). Supposé qu'un tel exercice d'expérimentation littéraire soit traduisible dans une langue, non pas dans un couple de langues, c'est accepter d'emblée que cette traduction ne préserve point l'intention auctoriale. En effet, dans l'édition française de 1984, seul l'aspect différencié des lettres (en romain et en italique) et une note de l'éditeur rappellent les données de début du projet de création. Dans la version française, un «bilinguisme d'écriture" (cf. Oustinoff 2001) qui se manifeste dans le même texte, donc un élément personnel, est sacrifié au profit d'une réception monolingue, un élément collectif et territorial. Ce ne sont que quelques-uns des aspects hors normes qui caractérisent ce livre et entravent sa réception.

\section{Du contrepoint à la fugue. Texte, hypotexte, hypertexte, intertexte}

Ce titre cognitif6 (Cuvântul nisiparniţă - 1994, 2005, Le Mot

\footnotetext{
5 Voir dans ce sens aussi l'expérience d'autotraduction et de traduction mentale de Fernando Pessoa (Jorge de Sena, Introduction à Fernando Pessoa. Poemas ingleses. Lisbonne : Atica, 1987: 13-14, cité par Oustinoff 2001, 47).
} 
sablier -1984; La parabola ampolleta - 2013), saisit la genèse d'un texte autoréflexif qui affirme l'idée du texte et celle de la textualité, et scrute également le roman pour confirmer son autoréférentialité, « le discours théorique [n'étant] qu'une acrobatie en marge du texte » (Cuvântul nisiparniţă 2005, 27).

Tout se passe dans la tête de l'écrivain. Le roman mental est (dé-), (re-) verbalisé, matérialisé tantôt dans un code, celui du roumain, tantôt dans un autre, celui du français. (Lungu-Badea 2009, 22). Bien que, dès les premières lignes, il semble que l'écrivain se conforme aux anciennes conventions du genre romanesque, le changement se produit sans avertissement. Les thèmes et les métathèmes s'exposent, s'opposent, s'entrechoquent : le récit de l'écrivain, le récit du traducteur, celui du tavernier, du cafetier, du soldat démobilisé par sa propre volonté. Explicites, implicites, actuels, graves, des thèmes tels que : l'exil géographique, l'exil linguistique, la quête identitaire, la démythisation du communisme, jouent le rôle de passerelles entre l'Orient et l'Occident, entre le passé et l'avenir de l'Europe ; ce sont les points forts d'un plaidoyer et d'un témoignage sur la diversité des structures narratives et sur le style biscornu de Tsepeneag (Lungu-Badea 2009, 23).

L'une des fins et significations d'une œuvre est d'être partagée aux autres et avec d'autres. À qui et avec qui Tsepeneag partage la joie de créer ? Gestes inégaux, cohérence idéique composite, écriture fragmentaire bilingue, mélanges d'écriture et de réécriture, tout cela se superpose comme sur un parchemin manuscrit dont les couches innombrables gardent, chacune, l'empreinte de l'écrivain (Genette 1982), un repère de ce qu'il est ou de ce qu'il semble/veut être : un condamné « à besogner toute la vie sur le même palimpseste » (Tsepeneag, Le Mot sablier). La création en soi et l'écriture paraissent être les préoccupations qui comptent pour l'écrivain, et donc son seul gain (Lungu-Badea 2009, 24).

Dans son hypertexte, ludique et subversif, Tsepeneag parodie aussi bien des œuvres qu'une pratique littéraire toute entière, qu'il parvient à déformer et transformer sans la défigurer. L'écrivain propose ainsi une littérature interactive, définissable par rapport à la tradition et aux canons littéraires qui constituent le terme de comparaison. La récusation des canons et la contestation des pratiques littéraires, l'abandon des traits communs aux textes

${ }^{6}$ Il était naturel, donc, que celui-ci soit préféré au titre descriptif : passerelle, pont vers le soi et vers les autres, un pont de la communication inter- et intralinguale. Nous avons déjà remarqué ailleurs le goût particulier de Tsepeneag pour les titres qui synthétisent les principes oniriques Arpièges. Rien ne sert de courir, par exemple. 
traditionnels (la ponctuation rigoureuse ${ }^{7}$, par exemple), le conduisent au texte polyphonique, où le rôle du narrateur est joué consécutivement par des identités narrataires multiples. Toutefois, les lecteurs peuvent déceler l'hypotexte et percevoir l'effet parodique qui vient compléter le tableau hypertextuel (cf. Genette 1982, 49) :

„iar în privința punctuației $i^{8}$ mai exact spus a lipsei de punctuație din anumite texte aşa-zis moderne [...] ar trebui să chibzuim foarte serios. ea poate dezorienta cititorul. şi dacă e să tragem o concluzie din asta

dar recunoașteți că și majusculizarea forțată de după fiecare punct ține de tiranie. de o tiranie a lecturii

impusă de autor

[...] de autor care-i dictează cititorului unde să se oprească: la punct numeri pană la trei, la virgulă spui unu

iar la punct și virgulă

și la două puncte $[\ldots]$

Să se oprească unde are chef el monsieur l'Auteur posesor printre altele și al adevărului ortografic (Cuvântul nisiparniță, 20-21)

quant à la ponctuation surtout à l'absence de ponctuation de certains textes dits modernes [...] on devrait réfléchir sérieusement. Elle peut désorienter le lecteur. et s'il faut en tirer une conclusion mais reconnaissez que la majusculation forcée qui suit chaque point relève de la tyrannie. une tyrannie de la lecture

imposée par l'auteur

[...] l'auteur qui ordonne au lecteur où s'arrêter. Au point on compte jusqu'à trois, à la virgule on dit un

mais au point-virgule

et aux deux-points [...]

s'arrêter là où a envie monsieur l'Auteur comme possesseur parmi d'autres de la vérité orthographique (Le Mot sablier)

en cuanto a la puntuación mejor dicho a la falta de puntuación de algunos textos así llamados modernos [...] tendríamos que reflexionar con seriedad. ella puede desorientar al lector. y si hay que sacar una conclusión de esto

pero tenéis que reconocer que la mayúscula forzada después de cada punto es signo de tiranía. una tiranía de la lectura

7 Les Noces nécessaires n'est point moins expérimental que Le mot sablier. Ce livre écrit sans ponctuation où l'espace blanc joue des rôles multiples de la ponctuation manquante a déterminé Jean Frémon à affirmer : "Que reste-t-il aujourd'hui de ce qu'on appelle le nouveau roman sinon la musique rauque de Claude Simon, le piano cristallin et nostalgique de Marguerite Duras, le pipeau familier de Robert Pinget..., c'est à ce niveau qu'il faut aujourd'hui placer Tsepeneag » (« Nouvelles littéraires », le 24 nov.-le $1^{\text {er }}$ déc. 1977).

8 Sorte de leitmotiv (Tepeneag, 2005, 15, 16, 17 x2, 20 x 2 et passim, nous soulignons). 
impuesta por el autor [...]

bueno en primer lugar por el autor que dicta al lector dónde se tiene que parar: al punto cuentas hasta tres. a la coma dices uno

y al punto y coma

y a dos puntos [...]

que se pare a donde le dé la gana Monsieur l'Auteur poseedor entre otras de la verdad ortográfica (La parabola ampolleta)

Grâce à ce prétexte de création, dans ce pré-texte, l'écrivain procède à la dé-formation du style qui l'a consacré. Après quatre-cinq avertissements « concernant la ponctuation9 » (Tsepeneag, Cuvântul nisiparniţă 2005, 21-22) et la critique (23), l'auteur décide de dévoiler au lecteur les règles de son jeu :

remplaçons la virgule: par les deux points parenthèses éventuellement même par tiret. le point joue aussi le rôle de virgule - dans certaines circonstances [remplaçons] le point également oui. et ne le mets plus à la fin du paragraphe où de toute façon il y a un espace blanc

l'espace (blanc) fait d'ailleurs partie de la ponctuation : si le point propose une pause, l'espace (blanc) l'impose (Cuvântul nisiparniţă 2005, 24)

Aux confins des règles de l'énonciation (Barthes 1984, 73), et irréductible à sa forme imprimée, l'hypertexte de Tsepeneag essaie de dépasser ces frontières. Mécontent des procédés rhétoriques et stylistiques en usage, il cultive une combinaison énonciative particulière, un double engagement et une double contrainte :

« astfel că s-ar cuveni înainte de toate să-i explic cititorului francez (o ce ipocrită reverență!) de ce încă îl mai privez de plăcerea textului direct: cel autentic şi concret în care ce-i drept n-aş mai avea scuza imperfecțiunii traducerii și nici pretextul că vezi doamne literatura română aflând-se la un alt stadiu de evoluție decât cea franceză (a fost săraca şi bolnavă de realism-socialist) cum dracu să mă lansez în alte și mai îndrăznețe aventuri scriptice » (Tepeneag, 2005, 7

«que j'explique au lecteur français (quelle hypocrite révérence) pourquoi je le prive du plaisir de lire le texte directement [en français]: le texte authentique et concret qui ne m'offrirait plus l'excuse de l'imperfection de la traduction ni le prétexte que la littérature roumaine se trouve dans une autre étape de son évolution que la littérature française, tiens, mon œil » (Le Mot sablier, traduit du roumain par Alain Paruit1984, 7)

9 La marotte de la ponctuation ne peut être que récurrente : « Suis-je vraiment obligé de mettre tout le temps des guillemets?... » (Pigeon vole, 105) 


\begin{abstract}
" así que convendría antes de todo explicar al lector francés (qué reverencia hipócrita) por qué sigo privándolo del gusto de leer un texto directo: el auténtico y concreto en el que es verdad que ya no tendría la excusa de la imperfección de la traducción ni el pretexto que la literatura rumana se encuentra en otro estado de evolución en comparación con la francesa (la pobre también ha estado enferma de realismo socialista) cómo demonios hago para lanzarme en otras aventuras de escritura aún más audaces » (Tsepeneag/Kovacs)
\end{abstract}

Le choix énonciatif reste toujours une convention, une entente entre l'auteur et le lecteur. Ce récit interactif s'articule à des niveaux différents - itératif, logique dramatique et énonciatif -, là où les consignes s'établissent. Le principe d'autogénération du texte est clair : « les mots dictent les mots ». Cet hypertexte n'existe que si l'on respecte le pacte d'écriture initial. Pour qu'il existe en tant que texte, la dualité s'impose impérativement: le discours s'adresse à quelqu'un. L'hypertexte se constitue de la sorte en tant qu'énonciation d'un discours, tout ce qui existe est situable : le drame de l'écrivain traduit, l'examen du cas de conscience du traducteur, le fossé creusé par les ouvriers, le fossé que l'écrivain saute, les leitmotiv, les mots qui viennent et s'en vont ( je ne connais pas le mot roumain et il est possible qu'il n'y en ait pas un qui dise toujours clepsydre ce qui serait une impropriété langagière, en tout il servait à faire bouillir les œufs »-Cuvântul nisiparniţă 2005, 10).

D'une cohérence organisationnelle apodictique, le fragment revendique le droit de nier ses origines. Le texte-rature de Tsepeneag préserve toutes les épreuves, les incessantes allées et venues. C'est un livre qui garde les biffures : le texte chantier dans le texte publié. Le fragmentaire devient ainsi l'enjeu de l'acte artistique, de l'acte de lecture et de l'acte de traduction et défie le lecteur à découvrir le mécanisme dont se sert l'écrivain pour engendrer un texte dans lequel s'agencent plusieurs romans autonomes, des textèmes, chacun véhiculant un sens. Ce «tout organique » et polyphonique construit autour de la relation de l'être humain au langage - se consolide grâce aux causeries, dans le café-taverne multifonctionnel, et aux prises de parole gérées par l'ingestion d'aliments (Cuvântul nisiparniţă 2005, 12, 16). Des notions pareilles à " commencement », « fin », " autonomie textuelle » témoignent de l'existence contingentée du texte, déterminée par l'existence d'un récepteur. En conséquence, c'est la textualité, et non pas l'écrivain, qui choisit le lecteur et le traducteur.

L'intertextualité a sa place dans Le Mot sablier. Dumitru Tsepeneag donne son nom à un texte, toutefois il le donne notamment à la relation qui se tisse entre le français et le roumain, à 
ce rapport artistique et expérimental qui fait l'objet de notre analyse. L'intertextualité et l'interlingual prétendent aussi bien la redéfinition des limites de ce texte, que la reconsidération de l'acte de création par le biais du bilinguisme de création: une poétique et une motivation de créer dans une langue d'adoption :

fiind călare pe două limbi mă hotărâsem să scriu în franceză. numai că a trebuit să constat cu destulă iritare şi ciudă că nu pot s-o fac până nu scap de fantasmele înmagazinate de-a lungul atâtor ani înc are în loc să scriu m-am gândit cum să scriu și la ce folos toți acești ani de așteptare în anticamera limbii franceze deci trebuie deocamdată să continui să scriu tot în română și în felul ăsta să mă debarasez în sfârșit de acest balast fantasmatic: căci cine îmi garantează că scriind în franceză nu mă trezesc bântuit de toate vedeniile astea cu de altfel s-a și întâmplat în câteva texte scurte șiatunci nu scriu ci descriu rescriu copiez ce n-am fost în stare să scriu dar mi-a rămas totuși în minte sub forma unor larve pe care nam cum să le evit. (Cuvântul nisiparniță, 8)

ainsi à cheval sur deux langues je m'étais résolu à écrire en français et ailleurs aussi dois-je continuer pour le moment à écrire en roumain pour me débarrasser enfin de tout ce ballast fantasmatique : car qui me garantit si j'écris en français que je ne me retrouverai pas hanté par tous ces spectres comme cela m'est déjà arrivé avec quelques brefs textes et dans ce cas je n'écris pas je décris je récris je copie ce que je n'ai pas été capable d'écrire mais ce qui est cependant resté dans mon esprit sous la forme de larves que je ne puis éviter (Le Mot sablier, 1984, 12, traduit par Paruit, en original en roumain).

de este reto y montado en dos idiomas me había decidido escribir en francés. pero he tenido que constatar con bastante irritación y pesar que no lo puedo hacer hasta que no deje atrás los fantasmas acumulados a lo largo de tantos años en los que en lugar de escribir he pensado en cómo escribir y a qué han servido todos estos años esperando en la antesala de la lengua francesa así que de momento tengo que seguir escribiendo en rumano y de esta manera deshacerme finalmente de este lastre fantasmático: porque quién me garantiza a mí que escribiendo en francés no me despierto embrujado de todos estos fantasmas como de hecho ya me ha pasado con algunos textos cortos así que no escribo sino que describo reescribo copio lo que no he sido capaz de escribir pero que sin embargo se me ha quedado en la mente como unas larvas que no tengo como evitar (La parábola ampolleta/Kovacs)

L'allusion à la littérature roumaine n'est qu'une stratégie textuelle qui donne le moyen de localiser l'intertexte/l'hypotexte. Une simple référence: Alain Paruit - le nom du traducteur de 
Tsepeneag et de nombreux écrivains roumains, traducteur auquel le livre est dédicacé - renvoie à des textes portant la signature du traducteur. Le fait d'évoquer le nom de son traducteur permet à l'écrivain de glisser son propre credo de traducteur :

«L'auteur traduit n'a aucune puissance, car aucune présence. Et comment être présent, sinon par les mots ? L'auteur est promis par la couverture, mais quand le livre s'ouvre, c'est un couvercle de cercueil qui se ferme. Ah ! bien sûr, cette mort est nécessaire ! Elle est même souhaitable. Ça fait partie des règles. Du jeu. » (Cuvântul nisiparniţă 2005, 68)

L'unité de ce textème découle des liens variables et structurels propres à l'ensemble de la création de Tsepeneag, et des rapports conjoncturels avec la littérature traditionnelle qu'il défie ici afin de se définir de façon quadrangulaire: 1) historiquement, par l'appartenance/la non appartenance à un courant; 2) selon la classe/le genre où il s'inscrit /refuse de s'y inscrire ; 3) du point de vue des canons littéraires qu'ils pourraient respecter/récuser, 4) du point de vue du style discursif modifié, par métamorphose/anamorphose.

L'intertextualité réclame la contextualisation extralinguistique et littéraire qui l'engendre : l'écrivain n'est pas à cheval que sur deux langues, mais également sur deux ou trois courants : l'onirisme esthétique et structural, les pratiques textuelles, le postmodernisme (Buciu 1998).

\section{Traduire l'écriture fragmentaire et hypertextuelle}

Plusieurs scénarios sont à prendre en considération que nous exposons sous la forme de questions :

- L'auteur aurait-il prévu /estimé que son public-cible ait des compétences linguistiques en français et en roumain ?

- Aurait-il présumé que ses lecteurs viennent des communautés bilingues, où le français est langue officielle à parité avec une autre langue, et dans de telles circonstances, à chaque traduction, on aurait pu/ pourrait préserver intacte la partie française (en original) et traduire dans une autre langue le texte écrit en roumain, en original (d'autres cas de figure en Lungu-Badea, 2011).

La version française, Le Mot sablier, est incontestablement autre chose que l'original Cuvântul nisiparniţă : une « métalepse du traducteur » (Saint-Gelais 2008, 7-18). Il serait absurde de reprocher 
au traducteur les contraintes d'écriture d'un texte original. Une traduction n'est qu'une traduction! (cf. Tsepeneag). Le Mot sablier s'engage dans un récit de l'expérience ontologique, de la création qui s'échafaude sur les supplices de l'écrivain jusqu'à ce que, vers la fin de l'ouvrage, le héros et le lecteur se rendent compte que c'est la création elle-même qui constitue le sujet du roman, à savoir l'histoire de la genèse d'un livre et de l'inspiration qui lui donne naissance.

Si la traductibilité est garantie par la thèse de la référentialité, son absence caractériserait logiquement l'intraduisibilité d'un texte. Les écrivains modernistes ont pratiqué l'illusion référentielle « de manière visiblement ironique » (Saint-Gelais 2011, 14).

La polyphonie du Cuvântul nisiparniţă/ Mot sablier n'admet pas une lecture prédéfinie, soumise à l'ordre canonique de la consécution accommodée que présentent les textes linéaires. Ce texte exige une lecture non linéaire, empruntant l'ordre auctorial. L'auteur suggère, d'ailleurs, de possibles itinéraires de lecture, chacun débouchant sur un texte potentiel et sur une lecture qui se soustrait aux standards rebattus, aux préjugés relatifs à la démarcation de la lecture linéaire. On observe ainsi que tout parcours textuel produit une nouvelle textualité : la condition du traducteur telle qu'elle est vécue par le traducteur même, la condition du traducteur telle qu'elle est décrite par l'auteur traduit, à son tour un traducteur chevronné, la condition du soldat démobilisé par sa propre volonté ou déserteur, selon la prise de vue, exilé sur le front, exilé en soi, etc. La technique de lecture hypertextuelle ne rend pas la compréhension facile, elle montre des carences quant à l'intelligibilité. De cette construction romanesque polyphonique même dérivent les hésitations du traducteur. De surcroît, la technique du contrepoint dénie l'existence d'un thème principal servant de référence aux autres, les thèmes renvoient alternativement les uns aux autres, devenant à tour de rôle le précédent des autres. Quelle méthode de traduction choisir pour transférer cette écriture fragmentaire?

Est-il difficile de traduire ce roman? Non. Au moins théoriquement, il ne devait pas l'être. Cependant, aux yeux de Tsepeneag, «deux cercles [vicieux ?] en forme de huit » (Cuvântul nisiparniţă 2005, 7) décrivent le rapport auteur- traducteur.

Si ce livre n'a pas été écrit pour être traduit, mais pour montrer comment une langue remplace une autre et rend, par la suite, inutile la traduction pour les usagers bilingues - francoroumains, alors c'est un plaidoyer pour la contre-traduction. Dans un tel cas de figure, on aurait dû le lire tel qu'il a été écrit. Cependant, vu que le roumain a longtemps été considéré comme une langue exotique, et vu même que le français n'est pas une langue pour tout le monde, l'acte de lecture eût été très peu probable. La traduction aurait été vaine dans le cas où le destin sociolinguistique du roumain 
avait été pareil à celui de l'anglais, comme le témoigne l'écrivain dans l'entretien avec Ion Simuţ (2003). On aurait pu alors traduire le livre dans toutes les communautés bilingues, ayant le français ou l'anglais comme langue officielle à parité avec une autre langue. Car, à chaque traduction, on aurait pu préserver intacte la partie française ou anglaise, ne traduisant dans l'autre langue officielle que le texte écrit dans la langue méconnue du public-cible. Vu ce destin hypothétique, ce livre expérimental, inédit au moment de sa production, aurait pu marquer définitivement les expériences textualistes, se configurant comme un repère intrinsèque. Trop de «si ». La réalité est bien loin des conjectures présentées. L'exotisme du roumain a limité la réception et l'influence potentielle de cette expérience littéraire sur d'autres écrivains. Les conditions n'étant pas requises, le texte n'est pas exempt de traduction.

\subsection{L'écriture fragmentaire comme unité de traduction, ou textème}

Aux pratiques de l'écriture fragmentaire qui métamorphosent les textes, viennent s'ajouter les métamorphoses traductionnelles. L'écriture fragmentaire bilingue subit donc une double métamorphose /anamorphose : celle que pratique l'auteur et celle que le traducteur est obligé de mettre en œuvre. Il s'agit de décider si l'on traduit vers une langue-cible ou vers un couple de langues-cible, à savoir :

- si l'on restitue les équivalences sémantiques, syntaxiques et morphologiques des deux langues-source du texte d'origine vers une seule langue-cible (comme en français) ;

- ou si l'on ne traduit que le roumain, langue maternelle et première langue-source, tout en choisissant de ne pas traduire la seconde langue-source, le français (comme on a pu constater dans la version espagnole).

Laquelle des deux manières de traduire souligne la potentialité des langues en relation de traduction? Laquelle est plus adéquate pour traduire une écriture fragmentaire bilingue, un textème (=unité de traduction insécable, non divisible sémantiquement) ?

À la traduction intralinguistique, mentale, et interlinguale (Lungu-Badea 2007, 72-86), tout comme dans le cas des traductions homophoniques, syntaxiques et homosémantiques des Oulipiens, le traducteur doit répondre fermement par l'identification et la maîtrise de la contrainte et, surtout, du public que vise l'œuvre à traduire. Ce n'est qu'ainsi qu'on peut espérer résoudre la «dialectique ricœurienne » concernant les deux maîtres du traducteur : l'auteur et le lecteur.

Traduire l'écriture autogénérationnelle de Tsepeneag qui, comme l'écriture oulipienne, c'est traduire de la même manière qu'on 
restitue la contrainte oulipienne, analyser les procédés de transformations textuelles et rendre (au moins ?) le principe de fonte, dé-fonte et refonte des textes, des idées. Parvenue ici, nous ne pouvons ignorer une question légitime: Le traducteur, doit-il faire preuve d'inspiration dans la traduction de l'écriture à contrainte ou suffit-il qu'il soit un bon technicien de la langue, un fabricant de textes selon des principes mathématiques?

\subsection{Motivations pour (ne pas) traduire la littérature expérimentale}

Les manières de création et les techniques de traduction ne sont pas toujours analogues (v. Tsepeneag, Paruit, Kovacs). Les limites de traduction de l'écriture fragmentaire bilingue sont données par :

- L'absence de finalité. Ce n'est que l'écriture qui compte;

- Le manque d'empathie entre l'auteur, le traducteur et le lecteur ;

- La polyphonie et la proche parenté de la traduction de ce texte et de la traduction de poésie par la prééminence de la forme au détriment du contenu. Le sens n'est pas pour autant étouffé parce qu'il naît au fur et à mesure que la pensée se matérialise. Les ratures ne sont que de façonnages d’idées, des bouts de phrases couchés sur papier.

- La difficulté de trouver un éditeur qui publie un livre de niche : la version espagnole définie en 2013 n'a pas encore trouvé un éditeur, ni un financement bien que l'écrivain se soit même impliqué dans ce projet.

\section{Conclusion}

Certainement, l'intertextualité et l'interlingual introduisent un nouveau mode de lecture, qui diffère bien sûr de la lecture linéaire: la lecture alternative. Serait-il insensé de parler de traduction alternative? Pourrait-on restituer l'écriture alternative tout en alternant avec les codes linguistiques du texte d'origine? La traduction semble être impossible, mais elle est à la fois nécessaire (cf. Derrida 1985). 
Annexe : Explicit libre

\begin{tabular}{|c|c|c|}
\hline $\begin{array}{l}\text { Je regarde par la } \\
\text { fenêtre le camion bleu } \\
\text { vert. Plus loin on voit } \\
\text { une tour. La pluie a } \\
\text { cessé. Le ciel } \\
\text { s'éclaircit. Les autres } \\
\text { ne s'en rendent pas } \\
\text { compte. Quoi que l'on } \\
\text { dise, je suis plus éveillé } \\
\text { qu'eux, plus près de la } \\
\text { fenêtre. Je regarde et } \\
\text { j’attends. Je ne sais } \\
\text { pas exactement quoi. } \\
\text { J'attends sans doute } \\
\text { que la bâche soit tirée } \\
\text { comme le rideau d'un }\end{array}$ & $\begin{array}{l}\text { Je regarde par la } \\
\text { fenêtre le camion bleu } \\
\text { vert. Plus loin on voit } \\
\text { une tour. La pluie a } \\
\text { cessé. Le ciel } \\
\text { s'éclaircit. Les autres } \\
\text { ne s'en rendent pas } \\
\text { compte. Quoi que l'on } \\
\text { dise, je suis plus éveillé } \\
\text { qu'eux, plus près de la } \\
\text { fenêtre. Je regarde et } \\
\text { j'attends. Je ne sais } \\
\text { pas exactement quoi. } \\
\text { J'attends sans doute } \\
\text { que la bâche soit tirée } \\
\text { comme le rideau d'un } \\
\text { théâtre. } \\
\text { Des marionnettes. } \\
\text { Sans fous et sans } \\
\text { cavaliers. Et que le } \\
\text { grand Guignol } \\
\text { commence! } \\
\text { En attendant je fouille } \\
\text { à nouveau dans mes } \\
\text { paperasses: "parlez- } \\
\text { vous français " répète } \\
\text { à voix basse le garde- } \\
\text { côte et fixe ses } \\
\text { jumelles sur l'horizon: } \\
\text { un avion qui ressemble } \\
\text { à un énorme oiseau } \\
\text { orange mais passe trop } \\
\text { vite et sort du champ }\end{array}$ & $\begin{array}{l}\text { Je regarde par la } \\
\text { fenêtre le camion bleu } \\
\text { vert. Plus loin on voit } \\
\text { une tour. La pluie a } \\
\text { cessé. Le ciel } \\
\text { s'éclaircit. Les autres } \\
\text { ne s'en rendent pas } \\
\text { compte. Quoi que l'on } \\
\text { dise, je suis plus éveillé } \\
\text { qu'eux, plus près de la } \\
\text { fenêtre. Je regarde et } \\
\text { j'attends. Je ne sais } \\
\text { pas exactement quoi. } \\
\text { J'attends sans doute } \\
\text { que la bâche soit tirée } \\
\text { comme le rideau d'un } \\
\text { théâtre. } \\
\text { Des marionnettes. } \\
\text { Sans fous et sans } \\
\text { cavaliers. Et que le } \\
\text { grand Guignol } \\
\text { commence! àn foulle } \\
\text { En attendant je fouille } \\
\text { à nouveau dans mes } \\
\text { paperasses: "parlez- } \\
\text { vous français" répète } \\
\text { à voix basse le garde- } \\
\text { côte et fixe ses } \\
\text { jumelles sur l'horizon: } \\
\text { un avion qui ressemble } \\
\text { à un énorme oiseau } \\
\text { orange mais passe trop } \\
\text { vite et sort du champ }\end{array}$ \\
\hline $\begin{array}{lr}\text { Țepeneag, } & \text { Cuvântul } \\
\text { nisiparniță, } & 2005 \\
\text { [1994]; en français en } \\
\text { original }\end{array}$ & $\begin{array}{l}\text { Tsepeneag/Paruit Le } \\
\text { Mot sablier (1984), en } \\
\text { français en original }\end{array}$ & $\begin{array}{l}\text { Tepeneag/Kovacs, La } \\
\text { palabra ampolleta } \\
\text { (2013), en français en } \\
\text { original }\end{array}$ \\
\hline
\end{tabular}

\section{Références bibliographiques}

BARNA, Nicolae. "Aller et venir à travers plusieurs goulots de sablier. Identité multiple, identité alternée, identité intégratrice ». In : G. LunguBadea, M. Gyurcsik (éds.). Dumitru Tsepeneag. Les Métamorphoses d’un créateur : écrivain, théoricien, traducteur. Timişoara, Editura Universităţii de Vest, 2006 : 9-23.

BARTHES, Roland. Le bruissement de la langue - Essais critiques IV, Paris, Seuil, septembre 1984 . 
BUCIU, Marin Victor. Ţepeneag. Ïntre onirism, textualism, postmodernism [Tsepeneag. Entre onirisme, textualisme, postmodernisme], Craiova, Aius, 1998.

DERRIDA, Jacques. «Des Tours de Babel ». In : Joseph Graham (ed.). Difference and Translation, Cornwell Press, $1985: 165-248$.

DERRIDA, Jacques. Le Monolinguisme de l'autre, ou La prothèse d'origine. Ed. Galilée, 1996.

DERRIDA, Jacques. Qu'est-ce qu'une traduction "relevante »? Paris : Editions de L'Herne. Extrait du Cahier de L'Herne Derrida, nº 83, 2005.

DIMISIANU, Gabriel. "L'Onirisme subversif », Seine et Danube. Dossier Le groupe onirique, Paris, Editions Paris- Méditerranée, 2005 : 21-28.

GENETTE, Gérard. Discours du récit. Paris : Seuil, 1972

GENETTE, Gérard. Palimpsestes : la littérature au second degré, Paris : Seuil, 1982.

HERMANS, Theo. "The Translator's Voice in Translated Narrative ", Target, 8:1, 1996: p. 23-48.

LADMIRAL, Jean-René. "Traduire la forme ? traduire les formes ?... », dans Nadia D'Amelio (dir.), La forme comme paradigme du traduire. Actes du colloque des 29-31 octobre 2008 de l'Université de Mons-Hainaut, Mons, Éditions du CIPA; 2009.

LADMIRAL, JeanRené. «Esquisses conceptuelles, encore ... ». In : Christine Raguet (éd.). Palimpseste. Traduire ou "vouloir garder un peu de la poussière d'or ». Hommages à Paul Bensimon. Paris : Presses Sorbonne Nouvelle, hors série (2006) : 131-144.

LADMIRAL,J. -R. et E. M. LIPIANSKY.La communication interculturelle. Paris : Armand Colin (Bibliothèque européenne des sciences de l'éducation), 1995

LARRIVÉE, Isabelle. La Littérarité comme traduction : Abdelkébir Khatibi et le palimpseste des langues. Doctorat Nouveau Régime sous la direction de Charles Bonn, Université Paris XIII (Paris Nord), U.F.R. des Lettres, des Sciences de l'Homme et des sociétés, 1994.

LUNGU-BADEA, Georgiana, «Les voix fédérées ou confédérées de l'auteur et du traducteur », RIELMA, $2011: 71-84$.

LUNGU-BADEA, Georgiana. D. Tsepeneag et le régime des mots. Écrire et traduire " en dehors de chez soi ». Timisoara : Editura Universitatii de Vest, 2009.

LUNGU-BADEA, Georgiana. "Sur le bilinguisme du soi. Fictionnalisation des actes d'écrire et de traduire ». Dialogues francophones, 14/2008 : 1926.

LUNGU-BADEA, Georgiana. "An (In)Visible Bridge: From Mental To Interlingual Translation. Reflections On Translating The Experimental Writings Of Dumitru Tsepeneag ». In: Sanda Badescu (ed.). From One Shore to Another. Reflections on the Symbolism of the Bridges. Cambridge Scholars Publishing, Cambridge, 2007 : 72-86.

LUNGU-BADEA, Georgiana. "Un minimaliste acharné: Dumitru Tsepeneag ». Dialogues francophones, $\mathrm{n}^{\circ} 12 / 2006$ : 199-209, traduit du roumain ( «Un minimalist înrăit: Dumitru Țepeneag », Orizont, nr. 10 (1465), Serie nouă, XVI, 20 oct. 2004: 4-5) par Andreea Gheorghiu. Timişoara : Editura Universităţii de Vest. 
MARAGNES, Daniel. "L'exil de la langue ». Derades archives $\mathrm{n}^{\circ} 2,2 \mathrm{e}$ semestre, 1998. URI: membres.lycos.fr/derades/exilsdelalangue.html. Dernière consultation : le 12 avril 2008.

OUSTINOFF, Michaël. Bilinguisme d'écriture et autotraduction: Julien Green, Samuel Beckett, Vladimir Nabokov. Paris : L'Harmattan, 2001.

SAINT-GELAIS, Richard. " La métalepse du traducteur : Tsepeneag, Paruit, Le Mot sablier». In: Dialogues francophones. Timişoara, Editura Universităţii de Vest, $\mathrm{n}^{\circ} 14$ (2008) : 7-18.

SAINT-GELAIS, Richard. Les Fictions transfuges. La fictionnalité et ses enjeux. Paris, Editions du Seuil, 2011.

TुEPENEAG, Dumitru, SIMUŢ, Ion. Clepsidra răsturnată. Dialog cu Ion Simuţ, [Le Sablier renversé. Entretiens avec Ion Simuţ], Editura Paralela 45, Piteşti, 2003.

\section{Textes de référence}

Dumitru TSEPENEAG,

Mot sablier, traduction d'Alain Paruit. Paris : P.O.L. Editeur, 1984. Cuvântul nisiparniță, 2e édition soignée et postfacée par de Georgiana Lungu-Badea. Timişoara: Editura Universităţii de Vest, 2005 [1994].

La palabra ampolleta, traduit du roumain par Iulia Kovacs. Université de l'Ouest de Timisoara. Projet de traduction- master 2; dirigé par G. Lungu-Badea et déroulé dans le cadre des recherches menées par le Centre d'études ISTTRAROM-Translationes. 2013.

Arpièges. Traduit du roumain par Alain Paruit. Paris: Gallimard, 1973 .

Les Noces nécessaires. Paris: Flammarion, 1977.

Nunţile necesare. Bucureşti: Editura Fundaţiei Cuturale Române, 1992; Bucureşti: Editura ALLFA, 1998.

Roman de gare. Paris: P. O. L. éditeur, 1985. 